\title{
Internal Kinematics of the most Luminous H II Regions in M100
}

\author{
N. S. P. Sabalisck ${ }^{1}$, M. Rozas ${ }^{1}$, J. E. Beckman ${ }^{1}$ and J. H. Knapen ${ }^{2}$ \\ ${ }^{1}$ Instituto de Astrofísica de Canarias, La Laguna, Spain 38200 \\ nss, mrozas, jeb@ll.iac.es \\ ${ }^{2}$ University of Herfordshire, Hatfield, UK \\ knapen@star.herts.ac.uk
}

\begin{abstract}
We have used the TAURUS Fabry-Perot mapping spectrometer on the William Herschel telescope (WHT) to produce a complete kinematic map of the disk of M100 in $\mathrm{H} \alpha$. Here we show how the internal velocity dispersion $(\sigma)$ of the principal emission components of the brightest regions varies with their $\mathrm{H} \alpha$ luminosity. The plot shows ample scatter, but an upper envelope in $\sigma$ is clearly linear (in the log-log plane) with a slope of $2 \cdot 6$, a result which agrees precisely with an earlier graph by Arsenault et al., who selected instead the regions of highest surface brightness. We show that this result, which differs from the conventional prediction from the virial theorem, is consistent with virialisation if the H II regions are density bounded, and thus offers evidence in support of the density bounding hypothesis for the most luminous regions in disk galaxies.
\end{abstract}

Keywords: ISM: H II regions - ISM: kinematics and dynamics — galaxies: ISM galaxies: spiral

\section{Observations}

The observations were made with the TAURUS II instrument, at the Cassegrain focus of the WHT, in May 1995. As M100 is so large, we required two data cubes whose region of overlap contained the galaxy centre. We had $0 \cdot 29^{\prime \prime} /$ pix, and velocity steps of $10 \mathrm{~km} \mathrm{~s}^{-1}$, under photometric conditions with seeing of $1^{\prime \prime}$. Calibration followed the standard method of Atherton and Taylor.

\section{Results}

Figure 1 (below) shows a set of spectra of intensity versus radial velocity from individual H II regions. From spectra of this type we computed the internal velocity dispersions of the principal components in intensity of all the regions measured (the brightest 200 out of 2000 identified in M100). These are shown plotted against $\mathrm{H} \alpha$ luminosity in Figure 1 $(t o p)$. We can see a broad scatter, but the upper envelope is clearly linear in the $\log -\log$ plot. Its slope is $2 \cdot 6 \pm 0 \cdot 3$, which agrees with the slope of $L$ versus $\sigma$ found by Arsenault, Roy \& Boulesteix (1990) for the regions of M100 with highest surface brightness. There is a $10 \%$ random error in each specific $\sigma$ value and a basic systematic error of about $2 \cdot 5 \mathrm{~km} \mathrm{~s}^{-1}$.

\section{Interpretation}

The wide scatter in the dispersions implies that in general the regions are not in virial equilibrium. We interpret the upper envelope as that of virialised regions, and the higher $\sigma$ values for the rest as due to dynamical injections from $\mathrm{OB}$ winds or even supernovae (the value of $80 \mathrm{~km} \mathrm{~s}^{-1}$ in one component is entirely consistent with observations of galactic, well resolved regions). The slope of the virial envelope is not 4 , as predicted by Terlevich \& Melnick (1981) because these brightest regions are density bounded (see Rozas, Beckman \& Knapen 1996; Beckman, Rozas \& Knapen 1998, present issue p. 83), so the $\mathrm{H} \alpha$ luminosity is a decreasing fraction of the full ionising luminosity of the region. (Project support: Grant PB94-1107; DGICYT).

Arsenault, R., Roy, J. R., \& Boulesteix, J. 1990, A\&A, 234, 23

Beckman, J. E., Rozas, M., \& Knapen, J. H. 1998, PASA, 15,83

Rozas, M., Beckman, J. E., \& Knapen, J. H. 1996, A\&A, 307,735

Terlevich, R., \& Melnick, J. 1981, MNRAS, 195, 839 

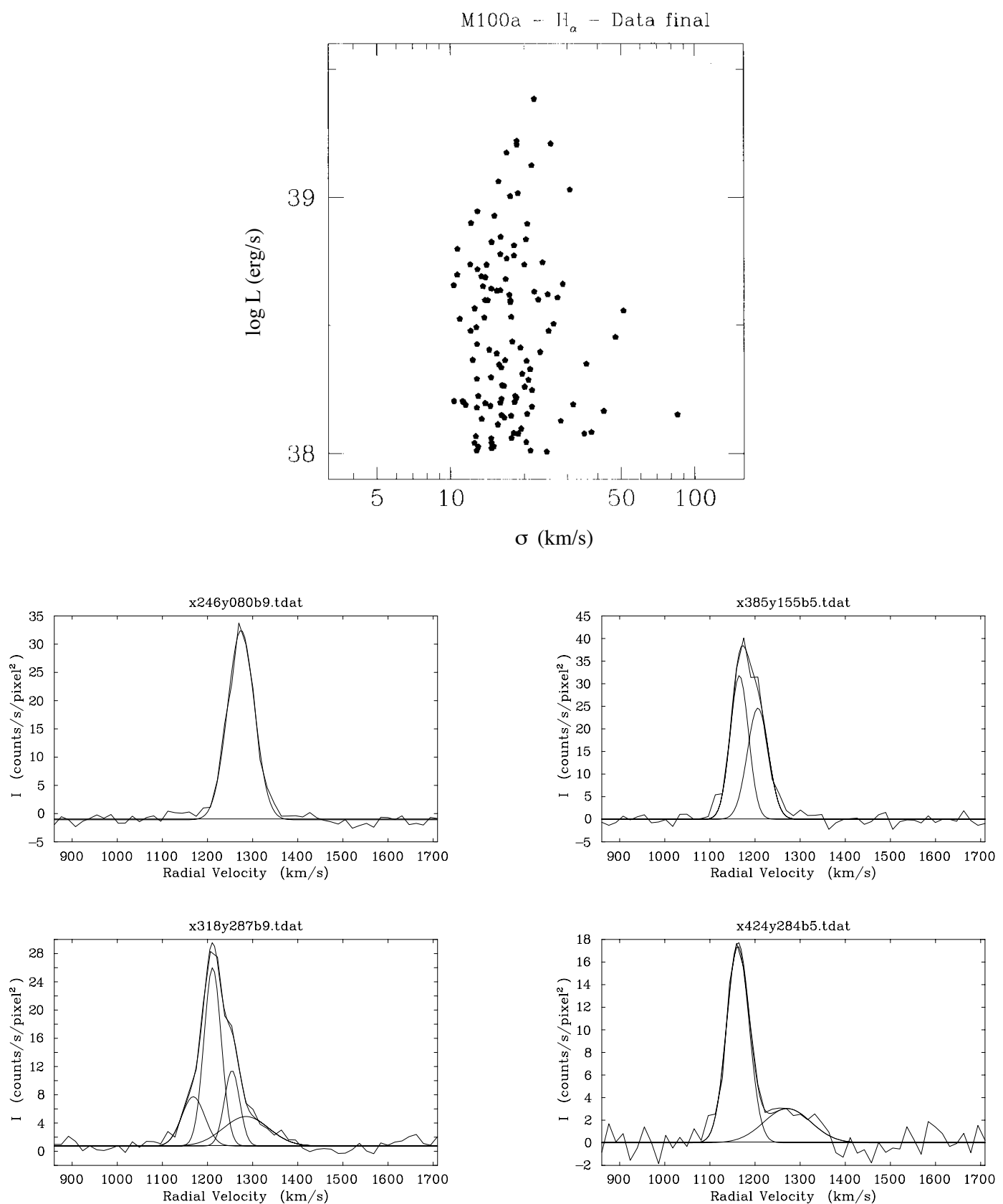

Figure 1-Top: Luminosity versus velocity dispersion $(\sigma)$ for the H II regions observed, using the principal component from each region. Values of $\sigma$ are corrected for instrumental and thermal broadening; values of $\sigma$ less than $10 \mathrm{~km} \mathrm{~s}^{-1}$ are not reliable, and are not included. Note the clean upper envelope (see text for its interpretation). Below: Emission line profiles from a selection of bright H II regions in M100, from the TAURUS map, with the individual kinematic components indicated. 